\title{
Análisis de las bronquiolitis por virus respiratorio sincitial hospitalizadas en un servicio de Pediatría durante dos temporadas separadas por 5 años y su relación con el broncoespasmo recurrente tras el alta
}

\author{
JÁ. Gómez Carrasco a, I. Merino Villeneuve ${ }^{\mathrm{b}}$, C. Herbozo Nory, \\ P. González Santiago ${ }^{a}$, G. López Loisa , E. García de Frías \\ ${ }^{a}$ Servicio de Pediatría, Hospital Príncipe de Asturias, \\ Departamento de Especialidades Médicas, Universidad de Alcalá. Madrid. España. \\ ${ }^{b}$ Pediatra. CS Fuencarral. Madrid. España.
}

\author{
Rev Pediatr Aten Primaria. 2009; I I:207-I8 \\ José Ángel Gómez Carrasco, joseangel.gomez@uah.es
}

\begin{abstract}
Resumen
Introducción: la bronquiolitis (BQL) es la infección de las vías respiratorias inferiores más frecuente en lactantes menores de 1 año y una causa importante de hospitalización. El virus respiratorio sincitial (VRS) es el agente causal en el 70\%-80\% de los casos. Nos hemos preguntado si, en nuestro medio, la variación en las diferentes tendencias terapéuticas a lo largo del tiempo se ha traducido en variaciones en los resultados clínicos.

Material y métodos: se incluyeron en el estudio todos los lactantes menores de 2 años (<24 meses) hospitalizados en nuestro servicio de Pediatría, por un primer episodio de BQL causado por el VRS, durante dos temporadas con un intervalo de 5 años (2000-2001 y 2005-2006). Se analizaron las diferencias epidemiológicas, clínicas y de tratamiento existentes entre estas dos temporadas.

Posteriormente, las familias de los pacientes ingresados durante la segunda temporada, fueron entrevistadas mediante encuesta telefónica para obtener información clínico-epidemiológica sobre la evolución de la enfermedad tras el alta.

Resultados: se incluyeron 110 pacientes, 56 de la primera temporada (2000-2001) y 54 de la segunda (2005-2006). La BQL por VRS en nuestra muestra afectó fundamentalmente a lactantes menores de 1 año con una edad media al ingreso de 4,8 meses, sobre todo varones $(65,5 \%)$ y con una época epidémica invernal (noviembre-febrero). En cuanto al tratamiento, destacó el menor uso de oxígeno adicional y de $\beta_{2}$-agonistas, y el mayor uso de fluidoterapia intravenosa y de adrenalina nebulizada durante la segunda temporada respecto a la primera.
\end{abstract}

Los autores declaran no presentar conflictos de intereses en relación con la preparación y publicación de este artículo. 
En el estudio de seguimiento, mediante encuesta telefónica, el 56,6\% de los pacientes entrevistados presentó algún nuevo episodio de broncoespasmo tras el alta hospitalaria, precisando asistencia en urgencias el 58,8\%, siendo estos porcentajes mayores en aquellos pacientes con antecedentes familiares de broncoespasmo.

Conclusiones: aunque el manejo de la BQL por VRS ha variado en estos 5 años, las variables principales, como son la estancia media hospitalaria (5,2 dias) y las complicaciones (intubación, traslado a la unidad de cuidados intensivos), no se han visto modificadas sustancialmente.

Destacar también, las consecuencias a largo plazo de la BQL por VRS al favorecer el desarrollo de hiperreactividad bronquial, siendo la existencia de antecedentes familiares de asma un importante factor predictor.

Palabras clave: Bronquiolitis, Virus respiratorio sincitial, Hiperreactividad bronquial, Antecedentes familiares.

\section{Abstract}

Introduction: bronchiolitis is the most frequent infection of the lower respiratory tract among infants under 1 year and a significant cause of hospitalization. Respiratory syncytial virus is the causal agent in $70 \%-80 \%$ of the cases. Our main goal was to check if different therapeutic tendencies over time have caused variations in clinical results.

Patients and methodology: the study includes infants under 2 years of age admitted to our Pediatrics Department after a first episode of respiratory syncytial virus bronchiolitis, during two periods separated by 5 years (2000-2001 and 2005-2006). Epidemiological, clinical and treatment differences between these two periods were analyzed.

Subsequently, the families of patients admitted to hospital during the second period were interviewed by phone in order to obtain clinical and epidemiological information about the evolution of the disease after hospital discharge.

Results: one hundred and ten patients were included; 56 from the first period (20002001) and 54 from the second (2005-2006). The respiratory syncytial virus bronchiolitis, in our sample, affected mainly to infants under 1 year, with an average age of 4.8 months when admitted, most of them were male (65.5\%) and during the winter season (November-February). Regarding treatment, during the second period additional oxygen and ,2 agonists were used less, while the use of intravenous fluid-therapy and nebulized adrenaline increased.

During the follow-up study made by phone interviews, $56.6 \%$ of the patients presented with a new wheezing episode after hospital discharge, $58.8 \%$ of them requiring healthcare assistance; these patients had a higher family history of asthma.

Conclusions: even though the treatment of bronchiolitis has changed during these 5 years, main variations such as average hospital stay (5.2 days) and complications (intubations, referral to the ICU) did not have any significant modifications.

It is also important to highlight the long term consequences of bronchiolitis derived from respiratory syncytial virus, because they can cause bronchial hypereactivity. A family history of asthma is considered a significant predictor of recurrent wheezing episodes.

Key words: Bronchiolitis, Respiratory syncytial virus, Bronchial hyperreactivity, Family history. 


\section{Introducción}

La bronquiolitis (BQL) es la primera causa de hospitalización en niños durante el primer año de vida, con unas tasas de 31 por 1.000 , y representa una gran proporción del gasto sanitario en este grupo de edad. Conceptualmente la BQL se define como un trastorno debido a la infección viral de las vías respiratorias inferiores, que frecuentemente afecta a lactantes ( $<2$ años), y se caracteriza por: inflamación aguda, edema y necrosis de las células epiteliales que revisten las pequeñas vías aéreas, lo que se traduce en un incremento en la producción de moco y broncoespasmo. Esta definición de BQL incluye la afectación histológica de las pequeñas vías respiratorias, por lo que en la clínica mayoritariamente los pediatras consideramos que estamos ante una bronquiolitis cuando concurre una constelación de síntomas y signos que incluyen: pródromos de vías respiratorias altas (rinorrea), seguidos de signos de afectación de vías respiratorias inferiores: esfuerzo respiratorio y "jadeo" (tos, crepitantes, sibilantes, taquipnea, esfuerzo respiratorio, retracciones, etc.), en menores de 2 años ${ }^{1-3}$. Es la infección de vías respiratorias inferiores más frecuente en lactantes menores de 1 año y es la causa más frecuente de hospitalización de los lactantes menores de 6 meses $^{4}$. La incidencia alcanza su máximo en invierno y al comienzo de la primavera. En España, en epidemia invernal (noviembre-marzo), la incidencia es del $10 \%$ de lactantes y un $15-20 \%$ requieren ingreso hospitalario ${ }^{5}$.

La BQL aguda es preferentemente una enfermedad vírica. Aunque el virus respiratorio sincitial (VRS) es el agente causal en el $70-80 \%$ de los casos, otros virus como los parainfluenza, adenovirus, metaneumovirus $y$, en ocasiones, los micoplasmas, pueden ser responsables del resto de los casos $^{6}$.

El diagnóstico de BQL es fundamentalmente clínico y el tratamiento medicamentoso es controvertido. La elevada demanda asistencial, el elevado número de hospitalizaciones y las consecuencias a largo plazo, nos han impulsado a realizar este estudio centrado fundamentalmente en las BQL por virus respiratorio sincitial. En primer lugar, nos hemos preguntado si, en nuestro medio, la variación en las diferentes tendencias terapéuticas a lo largo del tiempo se ha traducido en variaciones en los resultados clínicos. En segundo lugar, y puesto que la BQL por virus respiratorio sincitial parece estar asociada al desarrollo de asma, hemos entrevistado a algunas familias de los pacientes hospitalizados en 
nuestro centro durante la segunda temporada mediante encuesta telefónica, para obtener información clínico-epidemiológica de la evolución de la enfermedad en los meses posteriores al alta.

\section{Material y métodos}

Estudio descriptivo sobre epidemiología, clínica y tratamiento de la BQL por VRS en pacientes hospitalizados en nuestro Servicio de Pediatría del Hospital Príncipe de Asturias (Alcalá de Henares), durante dos temporadas con un intervalo de 5 años: 2000-2001 y 2005-2006. El presente estudio incluye 110 pacientes, 56 de la primera temporada (20002001) y 54 de la segunda (2005-2006). Se trata de una muestra homogénea; todos los pacientes incluidos en nuestro estudio son lactantes ( $\leqslant 24$ meses) que han ingresado por un primer episodio de BQL por VRS. El antígeno del VRS se detecta en una muestra de frotis nasofaríngeo mediante la técnica de inmunoanálisis enzimático (ELISA). Todos los datos recogidos en este estudio están reflejados en el $100 \%$ de los casos en las gráficas de enfermería y son: año y mes del ingreso, sexo, edad en meses del paciente, días de estancia, necesidad o no de oxígeno y días de oxígeno, necesidad o no de $\beta_{2}$-agonistas nebulizado, de adrenalina nebulizada, de corticoides orales, de antibióticos oral o parenteral y de fluidoterapia intravenosa, presencia o no de dificultad respiratoria, de apneas, de tos intensa, de rechazo de tomas de fiebre (temperatura mayor a $38^{\circ} \mathrm{C}$ ), necesidad o no de intubación y de traslado a la unidad de cuidados intensivos [UCI]). Se tuvo constancia de la práctica o no de una radiografía de tórax en 106 de los 110 pacientes; solo en cuatro casos $(3,6 \%)$ no se reflejó este dato en la historia. Se realizó posteriormente el análisis de las diferencias epidemiológicas, clínicas y del tratamiento existentes entre esas dos temporadas.

En una segunda fase, las familias (padre o madre) de los pacientes ingresados durante la segunda temporada, fueron entrevistadas mediante encuesta telefónica para obtener información clínico-epidemiológica sobre la evolución posterior de la enfermedad. El tiempo medio transcurrido desde el ingreso hasta la entrevista fue de 14,5 meses. Se analizan variables dirigidas a describir rasgos atópicos y de hiperreactividad bronquial post-BQL VRS positiva: edad en meses del paciente en el momento de la entrevista, sexo, posteriores episodios de broncoespasmo, visitas a urgencias hospitalarias, ingresos generados por estas patologías, si ha recibido o no tratamiento preventivo y cuál, si ha precisado uso de corticoides orales, desa- 
rrollo de dermatitis atópica y de alergia alimentaria, si acude a guardería, presencia y número de hermanos mayores y si tiene antecedentes familiares de asma y/o broncoespasmo.

De las 54 familias con intención de entrevistar, se obtuvo información en 30. De las 24 restantes, 1 no había indicado su número de teléfono, en 5 el número de teléfono recogido era erróneo y en 18 no se obtuvo respuesta a la llamada tras tres intentos realizados en días y horas distintas.

Para los análisis estadísticos descriptivos se ha utilizado el paquete estadístico SPSS ${ }^{\oplus}$ versión 14.0. Para comparar proporciones, se ha utilizado la prueba de la chi cuadrado. Para comparar medias, se ha utilizado la prueba de la $t$ de student independiente. El nivel de significación se ha establecido con un error alfa de 0,05.

\section{Resultados}

Estudio de 110 pacientes, 56 de la primera temporada (2000-2001) y 54 de la segunda (2005-2006), que ingresan por un primer episodio de BQL VRS positiva.

La edad media de los pacientes al ingreso fue de 4,8 meses (la edad mínima es de 0,25 y la máxima de 24 meses). La mayoría de los pacientes estudiados tu-

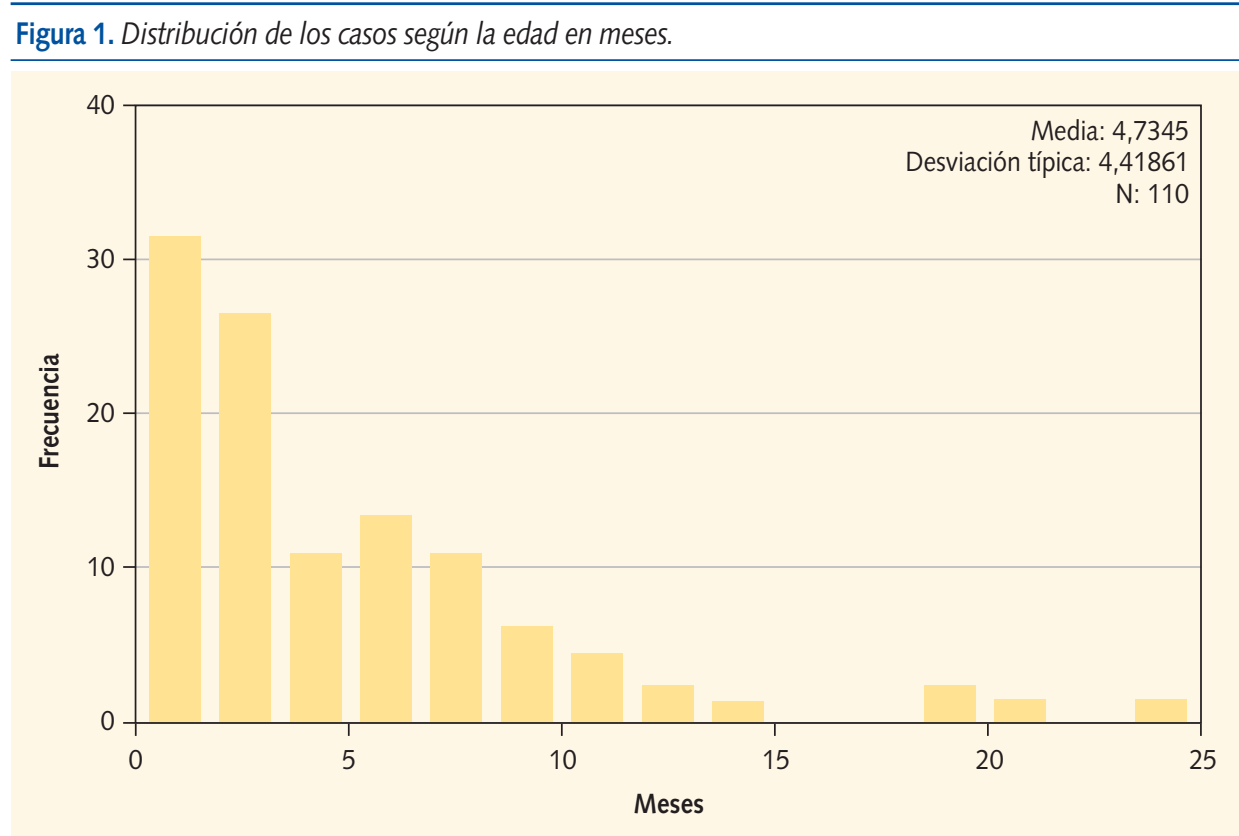


vieron menos de 12 meses cuando ingresaron por primera vez por una BQL VRS positiva (figura 1). La mayoría de los ingresos se concentran en los meses de invierno (noviembre-febrero), destacando los meses de diciembre $(41,8 \%)$, enero $(28,2 \%)$ y noviembre $(19,1 \%)$.

El $65,5 \%(72 / 110)$ de nuestros paciente fueron varones. La estancia media en el hospital fue de 5,2 días, no se encontraron diferencias estadísticamente significativas entre la primera $(5,52$ días) y la segunda (4,87 días) temporada de vigilancia $(p>0,05)$ (tabla I).

Las medidas terapéuticas de soporte básico en nuestros pacientes durante el ingreso fueron esencialmente oxígeno adicional (instauramos oxigenoterapia con valores de saturación de oxígeno menor o igual a $93 \%$ ) y fluidos intravenosos para mantener el estado de hidratación adecuado. El 62,7\% (69/110) precisó oxígeno adicional, con una me- dia de 3 días. De los pacientes de la primera temporada necesitaron oxígeno un $73,2 \%(41 / 56)$, frente al $51,9 \%$ $(28 / 54)$ de la segunda temporada $(p=$ 0,02 ). Precisó fluidoterapia intravenosa el 43,6\% (48/110) de los pacientes ingresados, siendo más empleada durante la segunda temporada $66,7 \%(36 / 54)$ que durante la primera $21,4 \%(12 / 56)$, $(p=0,01)$ (tabla I).

Respecto al tratamiento medicamentoso-sintomático de la BQL durante el ingreso, hemos observado que en el $63,6 \%(70 / 110)$ de los casos se emplearon $\beta_{2}$-agonistas nebulizados, en un $39,1 \%(43 / 110)$ adrenalina nebulizada y solo en el 17,3\% (19/110) corticoides orales. Comparando las dos temporadas, destacamos el mayor uso de $\beta_{2-}$ agonistas durante la primera temporada: $85,7 \%(48 / 56)$ versus $40,7 \%(22 / 54)$ en la segunda. Ello contrasta con el mayor uso de adrenalina durante la segun-

Tabla I. Principales diferencias en el manejo terapéutico de las bronquiolitis VRS+ entre temporadas y estancia media

\begin{tabular}{lccc}
\hline & $\begin{array}{c}\text { Temporada } \\
\mathbf{2 0 0 1 - 2 0 0 2}\end{array}$ & $\begin{array}{c}\text { Temporada } \\
\mathbf{2 0 0 5 - 2 0 0 6}\end{array}$ & $\begin{array}{c}\text { Nivel de } \\
\text { significación } \\
(\mathrm{p}<\mathbf{0 , 0 5})\end{array}$ \\
\hline Recibieron oxígeno & $\mathbf{7 3 , 2 0 \%}$ & $51,90 \%$ & $\mathrm{p}=0,02$ \\
Recibieron fluidoterapia & $21,40 \%$ & $66,70 \%$ & $\mathrm{p}=0,01$ \\
Recibieron $\beta_{2}$-agonistas adrenérgicos & $85,70 \%$ & $40,70 \%$ & $p=0,01$ \\
Recibieron adrenalina nebulizada & $1,80 \%$ & $77,80 \%$ & $p=0,01$ \\
Estancia media hospitalaria & 5,52 días & 4,87 días & $p>0,05$ \\
\hline VRS: virus respiratorio sincitial. & & & \\
\hline
\end{tabular}


da temporada: $77,8 \%(42 / 54)$ versus $1,8 \%(1 / 56)$ en la primera, siendo estas diferencias estadísticamente significativas $(p=0,01)$ (tabla I). En cuanto al uso de corticoides, no se observaron diferencias entre las dos temporadas. Cabe destacar que la edad media de los pacientes en los que se usaron los corticoides orales fue de 9,27 meses frente a los 3,89 meses de edad media de los pacientes en los que no se han usado corticoides $(p=0,01)$.

Un total de $44 / 110$ lactantes (40\%) recibió tratamiento antibiótico oral o parenteral durante su estancia hospitalaria, siendo el uso de antibióticos mayor durante la primera temporada $(65,9 \%)$ con una $p=0,01$. En el 55,5\% (61/110) de los pacientes se registró una temperatura axilar mayor de $38{ }^{\circ} \mathrm{C}$. De todos los pacientes que recibieron antibióticos (44/110), el 86,4\% (38/44) tuvo en algún momento una temperatura axilar mayor de $38{ }^{\circ} \mathrm{C}(p=0,01)$. De los 106 pacientes $(96,4 \%)$ de los que se tiene información respecto de la realización o no de exploración radiológica torácica, en $94(88,7 \%)$ se llevó a cabo dicha radiografía, siendo patológica en el 36,1\% (34/94). De todos los pacientes de los que se tienen datos acerca de la radiografía y que recibieron antibióticos (43/106), un $62,8 \%$ (27/43) tuvo ima- gen sugestiva de condensación y/o atelectasia en la radiografía de tórax ( $p=$ $0,01)$. En cuanto a la gravedad del proceso, solo el 4,5\% (5/110) presentó episodios de apnea durante el ingreso, ninguno precisó intubación (0/110) y solo un $1,8 \%(2 / 110)$ necesitó traslado a una $\mathrm{UCI}$ pediátrica.

El estudio de seguimiento mediante encuesta telefónica al padre o madre de los lactantes ingresados en la segunda temporada, se realizó tras un tiempo medio de 14,5 meses desde el ingreso. Solo se obtuvo información en el $55,5 \%$ (30/54) de los entrevistados. La edad media en meses de los pacientes en el momento de la encuesta era de 18,6 meses, con una mediana de 17,5 meses.

El $56,6 \%(17 / 30)$ de los pacientes encuestados había presentado algún nuevo episodio diagnosticado como bronquitis o broncoespasmo tras el alta hospitalaria. El 58,8\% (10/17) había acudido al servicio de urgencias del hospital por alguno de estos motivos con una media de seis visitas por persona y solo el $11,7 \%$ (2/17) precisó un nuevo ingreso generado por alguna de estas patologías. Habían recibido tratamiento preventivo del asma-broncoespasmo un 30\% (9/30) de los pacientes entrevistados, siendo los más prescritos los corticoides inhalados (budesonida, fluticasona) en un $66,7 \%$ 
(6/9) de los casos, seguidos de montelukast (Singulair) ${ }^{\circledR} 11,1 \%(1 / 9)$ y la asociación de corticoide más montelukast en un $22,2 \%(2 / 9)$.

Debido a la importancia de los contagios de infecciones virales en la etiopatogenia del broncoespasmo recurrente del lactante, interrogamos sobre la asistencia a guardería y/o la presencia de hermanos mayores en el domicilio: en el momento de la entrevista el $40 \%$ $(12 / 30)$ de los niños asistía a guardería y el $60 \%(18 / 30)$ tiene hermanos mayores con los que convive.

En cuanto al estudio de rasgos atópicos, el $16,7 \%(5 / 30)$ de los lactantes han presentado dermatitis atópica y solo el 3,3\% (1/30) alguna alergia alimentaria.

El $46,7 \%(14 / 30)$ tiene antecedentes familiares en primer grado de asma $y / 0$ broncoespasmo. El 78,5\% (11/14) de los pacientes con antecedentes familiares de asma y/o broncoespasmo presentaron episodios recurrentes de bron- quitis o broncoespasmo o asma, frente al 37,5\% (6/16) sin antecedentes familiares $(p=0,02)$. Destacar, a su vez, que los que tienen antecedentes familiares de asma y/o broncoespasmo, visitan más la urgencia, 57,1\% (8/14), que los que no tienen antecedentes familiares $12,5 \%(2 / 16)$, siendo la diferencia estadísticamente significativa $(p=0,01)$ (tabla Il y figura 2).

\section{Discusión}

Los resultados de este estudio demuestran que en la BQL por VRS cuando comparamos las dos temporadas analizadas, destaca un menor uso de oxígeno adicional y de $\beta_{2}$-agonistas y el mayor uso de fluidos intravenosos y adrenalina nebulizada durante la segunda temporada respecto a la primera. A pesar de estos cambios en el tratamiento, la estancia media hospitalaria $(5,2$ días) se mantuvo similar en la primera temporada (5,52 días) y en la segunda temporada (4,87 días). Resaltamos que

\begin{tabular}{|c|c|c|c|c|c|c|}
\hline \multirow[t]{2}{*}{ Antecedentes familiares } & & \multicolumn{2}{|c|}{ Sí } & \multicolumn{2}{|c|}{ No } & \multirow[b]{2}{*}{$\%$} \\
\hline & & $\mathrm{N}$ & $\%$ & $\mathrm{~N}$ & $\%$ & \\
\hline \multirow{2}{*}{$\begin{array}{l}\text { Episodios posteriores de } \\
\text { broncoespasmo tras la BQL }\end{array}$} & Sí & 11 & 64,7 & 6 & 35,3 & 100 \\
\hline & No & 3 & 23,1 & 10 & 76,9 & 100 \\
\hline \multirow[t]{2}{*}{ Visitas a urgencias por ello } & Sí & 8 & 80,0 & 2 & 20,0 & 100 \\
\hline & No & 6 & 30,0 & 14 & 70 & 100 \\
\hline
\end{tabular}


Figura 2. Antecedentes familiares de broncoespasmo y episodios posteriores de broncoespasmo tras la bronquilitis (expresado en proporciones) $(p=0,01$ ).

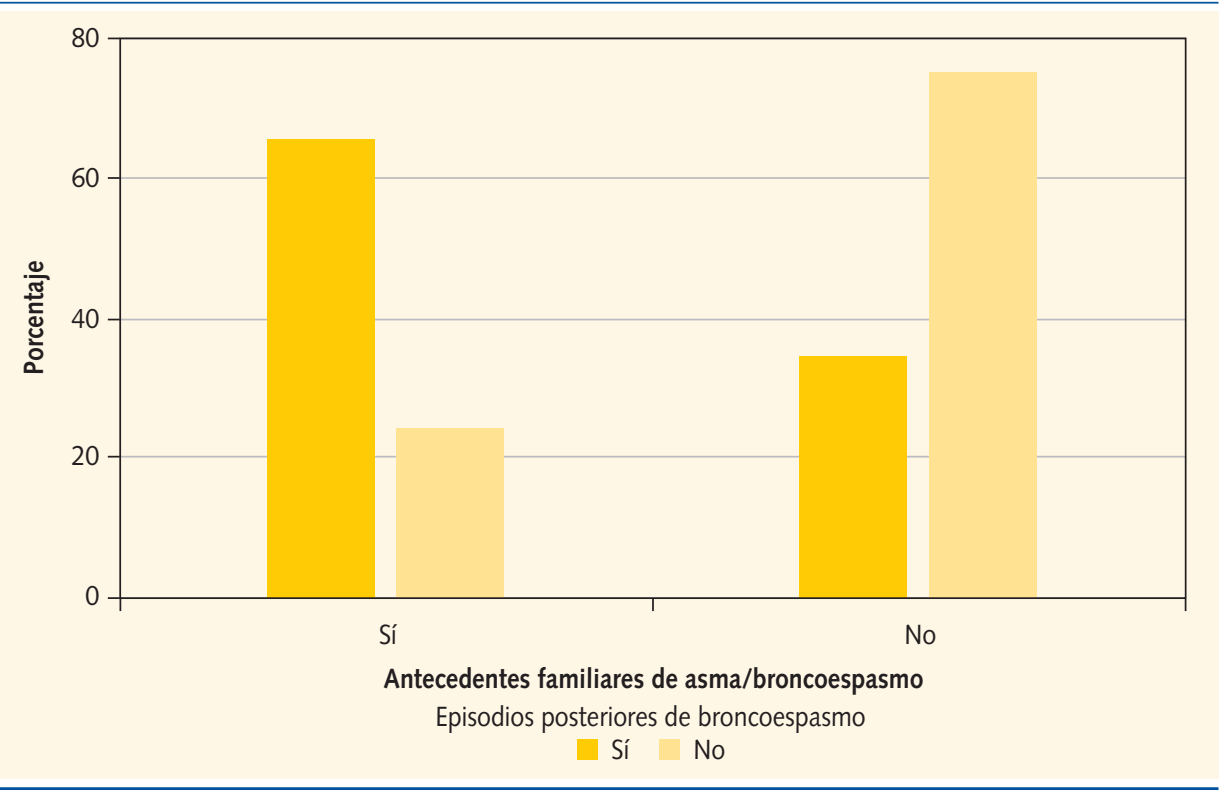

la edad media de los pacientes que recibieron corticoides orales fue de 9,27 meses.

Tres artículos recientes (2004-2005) exponen las controversias existentes respecto al tratamiento de la $B Q L^{3,4,7,8}$. En los tres, las conclusiones son muy similares. Preconizan que el único tratamiento eficaz de las BQL es la oxigenoterapia y la fluidoterapia intravenosa. Múltiples estudios clínicos aleatorizados han fracasado en su intento de demostrar la eficacia clínica (estancia hospitalaria, saturación de oxígeno, dificultad respiratoria) de otros tratamientos co- mo son los broncodilatadores, la adrenalina y los corticoides. Dado el coste, los efectos secundarios y la no eficacia demostrada de estos fármacos para el tratamiento de la $B Q L$, su uso rutinario y repetitivo no está justificado. No obstante y dado que los límites entre BQL, broncoespasmo recurrente y asma pueden resultar difusos, podría ser razonable en todos estos casos diagnosticados de $B Q L$ realizar un tratamiento "de prueba" con broncodilatadores $\mathrm{y} / \mathrm{o}$ adrenalina nebulizada, y suspender el tratamiento en caso de no ser considerado eficaz ${ }^{4}$. 
En el estudio de seguimiento mediante encuesta telefónica, destacamos que el $56,6 \%$ de los pacientes entrevistados presentó algún nuevo episodio de BQL o broncoespasmo o asma tras el alta hospitalaria, precisando asistencia en urgencias hospitalarias el $58,8 \%$, con una media de seis visitas por paciente, siendo hospitalizado por tal motivo tan solo el $11,7 \%$.

Nuestro estudio ha permitido objetivar que son principalmente aquellos pacientes con antecedentes familiares de asma y/o broncoespasmo los que tuvieron más episodios recurrentes posteriores $(78,5 \%)$ y visitan más la urgencia $(57,1 \%)$ frente a los que no tienen antecedentes familiares de asma (37,5\% y $12,5 \%$ respectivamente). Estos resultados corroboran la idea de que la BQL por VRS podría estar asociada con el posterior desarrollo de una hiperreactividad bronquial.

Muchos estudios confirman que las BQL VRS positivas predisponen a la recurrencia, a los cuadros repetidos de tos y sibilancias y que la incidencia de asma es superior entre los niños que han sufrido infección por VRS clínicamente aparente respecto al control, ${ }^{9,1,11}$. Virtualmente, todos los niños se infectan por VRS en los 2-3 primeros años (el $50 \%$ en el primer año) pero solo algunos ha- cen BQL clínica; siendo frecuente entre estos el desarrollo de broncoespasmo recurrente en los meses o años posteriores, es decir, el desarrollo de asma. Schauer y cols. ${ }^{12}$ comunicaron un mayor riesgo de sibilancias recurrentes y sensibilización alérgica durante los 12 meses siguientes al padecimiento de una bronquilitis severa. En The Tucson Children's Respiratory Study (TCRS) se ha demostrado un riesgo elevado de sibilancias frecuentes hasta la edad de 11 años respecto de los controles $(p \leqslant 0,01)$, riesgo que ya no fue superior a los 13 años; (inicialmente con 1.200 niños y finalmente con 900 seguidos durante 13 años ${ }^{13,14}$. Resumiendo, la infección de vías respiratorias bajas por VRS clínicamente significativa, padecida en los primeros meses de vida, es un importante factor para el desarrollo de asma y de sensibilización atópica. Queda por dilucidar si la BQL por sí misma promueve el ulterior desarrollo de broncoespasmo recurrente, o, en cambio, la BQL es un marcador de susceptibilidad genética para el asma o la inflamación recurrente de la vía aérea.

El estudio inicial dirigido a definir los factores predictivos de asma (The Original Asthma Predictive Index (API)) se basó en los datos del TCRS, con una importante muestra y un largo seguimiento 
orientado a evaluar la enfermedad respiratoria en más de 1.200 niños $^{15,16}$. Como resultado de estos estudios se obtuvo el índice predictivo del asma (IPA) para niños con broncoespasmo en los primeros 3 años de vida. Este índice califica como criterios mayores la historia parental de asma, la historia personal de dermatitis atópica y la sensibilización alérgica a, al menos, un aeroalérgeno y considera criterios menores el broncoespasmo no relacionado con resfriados, una eosinofilia periférica $>4 \%$ y la sensibilización alérgica a leche, huevos o frutos secos. En nuestro breve estudio hemos podido confirmar que fueron los lactantes con $\mathrm{BQL}$ relevante, que además tenían antecedentes familiares de asma, quienes tuvieron mayor probabilidad de broncoespasmo recurrente. Así, el 78,5\% (11/14) de los pacientes con antecedentes familiares de asma y/o broncoespasmo presentaron episodios recurrentes de bronquitis o broncoespasmo o asma, frente al $37,5 \%(6 / 16)$ sin antecedentes familiares $(p=0,02)$. También, entre los que tienen antecedentes familiares de asma y/o broncoespasmo, las visitas a urgencias por razón de broncoespasmo fueron más frecuentes entre aquellos que tenían antecedentes familiares de asma: 57,1\% (8/14), frente al 12,5\% (2/16) ( $p=$ 0,01).

\section{Conclusiones}

Los datos de este trabajo indican que los ingresos por BQL VRS positiva afecta fundamentalmente a lactantes menores de 12 meses, y que aunque el manejo clínico de la $\mathrm{BQL}$ ha variado en estos 5 años, las variables principales como son la estancia media hospitalaria y las complicaciones (intubación, traslado a UCl) no se han visto modificadas sustancialmente, corroborando estos resultados, las conclusiones de muchos artículos que defienden la no eficacia de los broncodilatadores, la adrenalina nebulizada y corticoides orales en el tratamiento de la BQL.

Destacamos que la BQL VRS positiva, clínicamente relevante, al favorecer el desarrollo de hiperreactividad bronquial, conduce a menudo a la presentación de episodios recurrentes de broncoespasmo. Es la existencia de antecedentes familiares (en primer grado) de broncoespasmo y/o asma un importante argumento predictivo del desarrollo de dicha hiperreactividad bronquial. En este subgrupo de pacientes el tratamiento preventivo post-BQL con corticoides inhalados y/o antileucotrienos podría minimizar el futuro desarrollo de broncoespasmo recurrente o asma del lactante. 


\section{Bibliografía}

1. American Academy of Pediatrics Subcommittee on Diagnosis and Management of Bronchiolitis. Diagnosis and management of bronchiolitis. Pediatrics. 2006;118:1774-93.

2. Villa Asensi JR, González Álvarez MI. Aspectos clínicos y diagnóstico diferencial de las sibilancias en el lactante. En: Villa Asensi JR (ed.). Sibilancias en el lactante. Madrid: Luzán 5 S.A. de Ediciones; 2005. p. 55-75.

3. Goodman D. Trastornos inflamatorios de las vías respiratorias de pequeño calibre. Bronquiolitis. En: Behrman RE, Kliegman RM, Jonson HB. Nelson, Tratado de Pediatría. 17. . edición. Madrid: Elsevier; 2004. p. 1415-7.

4. Fitzgerald DA, Kilham HA. Brochiolitis: assesment and evidence-based management. Clinical Update. MJA. 2004;180:399-404.

5. Scarfone RJ. Controversies in the treatment of bronchiolitis. Current Opin Pediatr. 2005;17:62-6.

6. Carballal G, Videla CM, Espinosa A, Savy V, Uez $O$, Sequeira MD, et al. Multicentered study of viral acute lower respiratory infections in children from four cities of Argentina, 1993-1994. J Med Virol. 2001;64:167-71.

7. Steiner RW. Treating acute bronchiolitis associated with RSV. Am Fam Physician. 2004;69:32530.

8. King VJ, Viswanathan M, Bordley WC, Jackman AM, Sutton SF, Lohr KN, et al. Pharmacologic treatment of bronchiolitis in infants and children: a systematic review. Arch Pediatr Adolesc Med. 2004;158:127-37.

9. Sigurs N, Bjarnason R, Sigurbergsson F, Kjellman $B$. Respiratory syncytial virus bronchiolitis in infancy is an important risk factor for asthma and allergy at age 7. Am J Respir Crit Care Med. 2000;161:1501-7.

10. Stein RT, Sherrill D, Morgan WJ, Holberg CJ, Halonen $M$, Taussig LM, et al. Respiratory syncytial virus in early life and risk of wheeze and allergy by age 13 years. Lancet. 1999;354(9178):541-5.

11. Sigurs $N$, Gustafsson PM, Bjarnason $R$, Lundberg F, Schmidt S, Sigurbergsson F, et al. Severe respiratory syncytial virus bronchiolitis in infancy and asthma and allergy at age 13. Am J Respir Crit Care Med. 2005;171:137-41.

12. Schauer U, Hoffjan S, Bittscheidt J, Kochling A, Hemmis S, Bongartz $S$, et al. RSV bronchiolitis and risk of wheeze and allergic sensitisation in the first year of life. Eur Respir J. 2002;20:1277-83.

13. Taussig LM, Wright AL, Morgan WJ, Harrison HR, Ray CG. The Tucson Children's Respiratory Study. I. Design and implementation of a prospective study of acute and chronic respiratory illness in children. Am J Epidemiol. 1989;129: 1219-31.

14. Wright AL, Taussig LM, Ray CG, Harrison HR, Holberg CJ. The Tucson Children's Respiratory Study. II. Lower respiratory tract illness in the first year of life. Am J Epidemiol. 1989;129:1232-46.

15. Guilbert TW, Morgan WJ, Zeiger RS, Bacharier LB, Boehmer SJ, Krawiec M, et al. Atopic characteristics of children with recurrent wheezing at high risk for the development of childhood asthma. J Allergy Clin Immunol. 2004;114:1282-7.

16. Martinez FD, Wright AL, Taussig LM, Holberg CJ, Halonen M, Morgan WJ. Asthma and wheezing in the first six years of life. The Group Health Medical Associates. N Engl J Med. 1995; 332:133-8. 